\title{
Multilinguales
}

\section{Le style libertin de François-Antoine Chevrier}

François-Antoine Chevrier's Libertine Style

\section{Luisa Messina}

\section{OpenEdition}

Journals

Édition électronique

URL : http://journals.openedition.org/multilinguales/1277

DOI : $10.4000 /$ multilinguales. 1277

ISSN : 2335-1853

\section{Éditeur}

Université Abderrahmane Mira - Bejaia

\section{Édition imprimée}

Date de publication : 1 décembre 2014

Pagination : 134-150

ISSN : 2335-1535

\section{Référence électronique}

Luisa Messina, «Le style libertin de François-Antoine Chevrier », Multilinguales [En ligne], 4 | 2014, mis en ligne le 01 décembre 2014, consulté le 10 décembre 2020. URL : http://journals.openedition.org/ multilinguales/1277 ; DOI : https://doi.org/10.4000/multilinguales.1277

Ce document a été généré automatiquement le 10 décembre 2020.

\section{(c) (i) (9)}

Multilinguales est mise à disposition selon les termes de la Licence Creative Commons Attribution Pas d'Utilisation Commerciale - Pas de Modification 4.0 International 


\title{
Le style libertin de François-Antoine Chevrier
}

\author{
François-Antoine Chevrier's Libertine Style
}

\author{
Luisa Messina
}

1 François-Antoine Chevrier (1721-1762) est un écrivain français du libertinage, oublié aujourd'hui de la critique qui retient plus aisément les noms d'autres romanciers libertins de son siècle comme Choderlos de Laclos, Crébillon fils et Charles Pinot Duclos. Certes, de son temps, il se distinguait plutôt comme pamphlétaire. La notice que lui consacre F. Weil, dans la version électronique du Dictionnaire des journalistes (1600-1789), où il est admis en tant que correspondant littéraire, mentionne que "Chevrier n'a jamais ménagé qui que ce soit (...). Il eut des querelles avec la Comédie-Française (...).En 1758, à la suite de la publication de l'Histoire de Lorraine, il dressa contre lui le Parlement et le Bailliage de Nancy (...) ». Quant à sa carrière littéraire, marquée par la publication de satires telle que le roman Le Colporteur (1761), elle lui confère l'étiquette de libelliste.

Ses positions fortement polémiques, exprimées tantôt dans ses œuvres historiques, tantôt dans ses écrits libertins, ont marqué sa vie errante jusqu'à sa mort brutale, en 1762, à Rotterdam où il se serait réfugié pour échapper à la justice française.

Certains ont même établi une relation de cause à effet entre ses positions tranchées et son décès. Dans ses Mémoires (1808: 20-21), Favart, par exemple, soutient cette hypothèse. Chevrier aurait été tué sous l'ordre des gouvernants hollandais parce que sa mort aurait été nécessaire à imposer leur volonté aux Français ${ }^{1}$.

Par le biais de ce bref détour biographique, notre intention était d'expliciter un tant soit peu les raisons qui ont fait que la posture de polémiste de Chevrier a fait passer au second plan ses talents d'écrivain. Or, bien que Chevrier soit mort précocement, après une vie bien mouvementée, il a laissé une production littéraire riche, comprenant de nombreuses œuvres libertines. Celles-ci sont encore peu connues du grand public et de la critique. Nous tenterons de montrer qu'elles présentent pourtant tous les caractères de la littérature de libertinage. Mais, comme l'écrit fort justement Valentina Ponzetto : "Encore faut-il s'entendre sur la signification à donner aux termes "littérature libertine », de 
"romans libertins" ou de "romanciers libertins". Le XVIII ${ }^{e}$ siècle, on le sait, ignore cette terminologie » $(2007: 24)$.

Certes, cette catégorisation ne fait pas l'unanimité auprès de la critique parce qu'elle désigne un objet quelque peu hétérogène, comme le souligne Jacques Rustin (1978: 30), à propos du genre dominant de cette littérature qu'est le roman :

L'incertitude sur le roman libertin (...) vient tout naturellement des sens qui s'entrecroisent et s'excluent souvent mutuellement: ni roman polisson, ni roman rationaliste, ni roman aristocratique ou "féodal", ni roman poissard ou crapuleux, ni roman érotique, ni roman pornographique, il peut cependant revêtir tour à tour l'une de ces livrées; tantôt il véhicule une doctrine subversive (...), tantôt il reste à l'évocation des mours permissives ; tantôt il use de toutes les ressources d'un style libre (jusqu'à l'obscénité au besoin), tantôt au contraire il garde une retenue qui peut s'apparenter, dans certains cas, à l'extrême platitude. (Favart, $1808: 20-21)$.

Pour résoudre ce problème de classification, Jaques Rustin, mais il n'est pas le seul, propose d'étendre la définition proposée par H. Coulet $^{2}$, à l'endroit de Crébillon fils, à toute la littérature du libertinage :

Ne pourrait-on pas considérer comme libertin tout roman qui offre d'une façon ou d'une autre - quels que soit le style et les intentions problématiques de l'auteur - une peinture nécessairement partielle (et partiale) de l'univers du libertinage? (Rustin, $1978: 31$ ).

Nous optons donc, en ce qui nous concerne, pour la suggestion de ce chercheur et considérons la littérature du libertinage, simplement, comme la « peinture du libertinage ", à charge pour l'analyste d'un corpus d'étude choisi de déceler «les sens (qui) s'y entrecroisent (ou) s'excluent ».

\section{Chevrier, un écrivain libertin comme les autres}

2 Malgré la diversité des formes littéraires, le genre dominant le corpus littéraire libertin est sans aucun doute le roman de formation :

À l'intention pédagogique du narrateur qui prétend, par son exemple, se rendre utile aux générations montantes, répond, à l'intérieur du récit, l'apprentissage du héros. Il pénètre en ignorant dans un cercle dont les lois et les règles non écrites lui préexistent et qu'il lui faut assimiler. Pris en main par des instituteurs détenteurs des clés du code social, il est avant tout passif. Au début du moins, ce n'est pas lui qui pourchasse les femmes, mais celles-ci qui le recherchent. L'enseignement théorique ne suffisant pas, le débutant est mené à l'expérience par une femme plus âgée. (Trousson, 2001 : LVI-LVII)

La société, dans ce type de roman d'éducation, est celle de «la bonne compagnie ", (du) "le monde ", c"est-à-dire (...) le milieu de la vie sociale de l'aristocratie à Paris" (Montandon, 1999 : 303). La prédilection pour une telle forme est du reste facilement compréhensible puisque ce genre permet aux auteurs de mettre en évidence quelques moments significatifs (comme la découverte du corps et celle de l'autre sexe, le passage du désir à la possession physique) qui constituent les étapes fondamentales de l'éducation libertine. Cette préoccupation de ce type de roman implique que l'« on suiv(e) moins l'histoire d'une destinée individuelle, particularisée, qu'une sorte de schéma organisateur et un rituel» (Trousson, 2001: LVI-LVII). Cette dimension pédagogique de la littérature libertine a contribué à ce que ses auteurs soient accusés de corrompre les jeunes nobles et les esprits fragiles :

Une des principales causes de la débauche que l'on remarque aujourd'hui dans notre jeunesse, est la lecture de certains livres obscènes que quelques misérables auteurs répandent, de temps en temps, dans le public. Le nombre de ces infâmes ouvrages s'est extrêmement multiplié depuis quelques années. (Cit. par Wald Lasowski, 2005, p. XXV) 
De la même façon que les écrivains libertins de son époque, Chevrier convoque les éléments typiquement libertins, notamment : l'importance des récits de séduction, les passions hors mariage, la présence des acteurs sociaux tels les "petits-maitres » et «petites-maîtresses » intéressés par la recherche du plaisir, le dérèglement des mœurs dans les trois classes sociales françaises, la dérision du clergé jugé lascif, les lieux de rencontres amoureuses comme les cabinets et les "petites maisons", et Paris, décor principal des intrigues libertines.

Il est donc nécessaire d'analyser en détail les éléments qui distinguent le style libertin de cet auteur.

Premièrement, la production libertine de Chevrier se concentre sur le récit des liaisons adultères et passagères, en violation du mariage de raison, comme dans son roman Les Mémoires d'une honnête femme (1753), résumé en ces termes dans L'Année littéraire de novembre 1754 (Fréron, lettre IX) :

Une jeune femme nourrit dans son cœur une passion secrète pour un Chevalier de Malte, sachant qu'elle est à la veille d'épouser un autre amant. A peine est-elle mariée, qu'oubliant ce qu'elle doit à son mari, elle entretient un commerce illicite avec un jeune Duc, lui écrit des billets tendres, lui donne des rendez-vous, et lui offre des gages heureux de son amour. Un Vicomte se présente, elle renvoie le Chevalier, congédie le Duc, déteste le Mari, et devient éperdument amoureuse du Vicomte. Elle traite ce dernier comme les autres; il est remplacé par un quatrième; un cinquième lui succède, et leur fait éprouver à tous un sort funeste.

Deuxièmement, les écrits libertins de Chevrier relatent les liaisons amoureuses des "petits-maitres " ${ }^{3}$, jeunes et effrontés, comme Dorival (Amusements des dames), Genneville (Recueil de ces dames) et Ma-gakou (Ma-gakou) qui passent d'une amante à l'autre ; celles des jeunes filles libertines comme Bi-Bi (Bi-Bi), Miss Otwai (Mémoires d'une honnête femme), Belise et Mademoiselle Brillant (Le Colporteur); celles de femmes notables comme la comtesse de Norval (Le Quart d'heure d'une jolie femme) et Madame d'Erbigni (Le Colporteur) qui envisagent tout de même de sauver leur réputation.

Chevrier, comme les autres écrivains libertins, tourne en dérision le «petit-maitre » de son siècle. Voici la description qu'il en fait dans Les Ridicules du siècle (1752 : 24-25), au chapitre IV intitulé « Des Petits-Maitres »:

Le Petit-Maître du siècle est un homme qui joint à une figure avantageuse, un goût varié pour les ajustements; amateur de la parure, il doit marier agréablement l'agrément avec la magnificence; esclave de la mode et des préjugés du jour, il n'est point asservi à ces mots usés, follement consacrés parmi nous, sous les noms de raison et de vertu; copie exacte de la femme du grand monde, s'il differe d'elle, ce n'est que par un supplément d'extravagances et de ridicules; jaloux de plaire sans être amoureux, il cherche moins à être heureux que la gloire de le paraître; constant dans ses écarts, léger dans ses goûts, ridicule par raison, frivole par usage, indolent à flatter, ardent à tout anéantir, ennemi du public qu'il voudrait cependant captiver: rien à ses yeux n'est supportable que lui-même; encore craint-il quelquefois de se voir tenté, dans l'appréhension de se trouver moins aimable. Voilà le petitmaître de la première classe. Celui de la seconde n'est qu'une fade copie (...). ${ }^{4}$

Dans ses Mémoires sur les mœurs de ce siècle (1821), C.P. Duclos fait de même :

Il n'a pas de travers qui ne puisse être en honneur, et qui ne tombe ensuite dans le mépris. Tel a été le sort des petits-maîtres. On ne donna d'abord ce titre qu'à des jeunes gens de haute naissance, d'un rang élevé, d'une figure aimable, d'une imagination brillante, d'une valeur fine et remplie de grâces et de travers. Distingués par des actions d'éclat, dangereux par leur conduite, ils jouaient un rôle dans l'État, influaient dans les affaires, méritaient des éloges, avaient besoin d'indulgence et savaient l'art de tout obtenir. (Duclos, 1821 : 134-135) 
4 A l'instar des autres romanciers galants, Chevrier refuse de faire le portrait de la bourgeoisie et considère la vie à la campagne comme une alternative passagère à la vie mondaine parisienne :

La Comtesse (Belise), excédée de plaisir et d'esprit, prit le parti d'aller passer la belle saison dans une Campagne solitaire, où elle comptoit n'avoir avec elle que ses femmes et un jeune Abbé qu'elle avoit pris pour l'aider dans ses lectures. "Le projet de Belise fut exécuté sur le champ ; soitvuide dans le coeur, soit satiété des plaisirs, soit une lueur de raison, la Comtesse partit sans regretter Paris. On étoit alors dans les beaux jours du mois de Mai ; la nature animée respiroit partout la volupté: Belise alloit elle s'enfoncer dans un bosquet, pour y rêver sur les douceurs de la solitude, elle appercevoit, sans les chercher, deux jeunes moineaux, qui du plaisir passoient aux caresses, et des caresses revoloient aux plaisirs. (Chevrier, 1762/1914:25).

Le peu de place réservée au peuple, et surtout aux bourgeois, n'étonne pas si l'on admet, comme l'observe R. Démoris, que la hiérarchie littéraire se fonde sur la hiérarchie sociale :

Aux personnages nobles sont préparées les expériences les plus riches et complexes (telles qu'amour ou intérêts d'état), tandis que les thèmes de l'argent ou l'impact avec la réalité matérielle suggèrent le statut romanesque traditionnellement réservé aux bourgeois et aux gens à basse extraction sociale. (Démoris, $1971: 348$ )

Il est évident que les personnages séduisants de Chevrier sont surtout les représentants de la noblesse qui ne dédaignent pas d'avoir des intrigues momentanées avec des ecclésiastiques, des comédiens et des domestiques. En cela le roman Le Colporteur (1762/1914) est l'incarnation de cette promiscuité sociale :

- Mais mon serrurier et le laquais de Monsieur, reprit la Marquise, n'étant pas dans ce cas, pourquoi ces animaux-là jouent-ils? - Pour singer les grands, Madame, répliqua Brochure, et se donner un air. Vous sçavez (savez) qu'à Paris rien n'est si arrogant que le petit peuple et la Valetaille; ce sont exactement les Arlequins de la société, dont la fureur balourde est de parodier tout: on va aux Tragédies qu'ils jouent, comme aux Parades du Boulevard, où l'on met sa raison à l'écart pour s'amuser davantage. (p. 105)

Mais, Chevrier ne veut pas se focaliser sur la noblesse vivant à la cour parce qu'elle serait déjà trop connue du public : «Ce serait ici le lieu de peindre la cour ; mais le portrait est si usé, que vous me dispenserez de le renouveler à vos yeux» $(1787: 6)$.

En réalité, la fiction occupe moins de place qu'il n'y paraît dans les intrigues libertines peintes par Chevrier. Celles-ci sont inspirées de la réalité et couvrent toutes les couches sociales. En effet, les protagonistes sont des aristocrates lascifs (Le Colporteur), des danseuses et comédiennes du théâtre (Recueil de ces dames) avec lesquelles Chevrier est en contact en raison de son activité de dramaturge (Le Colporteur), des nobles de la Belgique (Les Amusements des dames de $B^{* * *}$, Les trois $C^{* * *}$ ), des religieux corrompus et luxurieux (La Vie du fameux père Norbert ex capucin). À ce propos, H. Coulet explique que le libertinage était une conduite élitaire reposant sur le privilège de classe (Coulet, 1978: p. 182). Mais Chevrier fournit en quelque sorte une explication dans Les Observations sur le théâtre (1755: 38-39) :

Un autre motif qui a pu déterminer les auteurs à ne mettre que des Marquis et des Comtesses sur le Théâtre, c'est qu'ils vivent avec eux, et que leurs écarts les frappent de plus près que ceux du Peuple qu'ils ne voient que de loin, rien n'a fait tant d'honneur à la haute Noblesse que de voir les gens de Lettres comme compagnie, elle a prouvé par là qu'elle frondait les préjugés d'ignorance dans lesquels elle a été si longtemps en France (...).

Cette réflexion pourrait être placée au fondement d'une étude sur la réception de la littérature du libertinage en ce qu'elle renseigne sur son lecteur et son horizon d'attente. 


\section{La satire des femmes dans les textes de Chevrier}

L'un des éléments les plus constants de la production de Chevrier est certainement la dérision réservée au personnage du libertin issu de l'aristocratie. Dans Les Ridicules $d u$ siècle (1752), Chevrier fait un portrait très détaillé du libertin à la conduite ridicule et lascive en se servant de l'italique pour souligner les expressions à la mode. Après avoir séduit la femme aimée, le libertin devient tyrannique et soucieux d'accroître sa réputation dans l'intention de susciter l'admiration des hommes et de déshonorer de nombreuses femmes qui glorifient ses entreprises.

Un homme du jour parvient-il par le secours des mines et des agaceries, à subjuguer une femme dont il a envie; il en devient bientôt le tyran. Un indiscret était autrefois un homme perdu; en voulant déshonorer sa Maîtresse, il se flétrissait lui-même: maintenant c'est un agréable, qui a l'heureux talent d'établir sa réputation sur le débris de celles des femmes; on le fête, on le recherche, oracle des sociétés, orateur de ce fantôme appelé la bonne compagnie, les hommes l'admirent, et le sexe, le dirai-je .... le sexe a l'imprudence de lui applaudir; faiblesse plus grande que celle même de céder (...). (Chevrier, 1752 : 7-8)

Ainsi, la femme est fortement présente dans la vie et la production de Chevrier. Alors que Chevrier manifeste un goût prononcé pour les femmes, il leur témoigne une détestation qui n'a jamais diminué. Il est possible que l'origine de ce sentiment contradictoire remonte au rapport que Chevrier avait avec ses deux sœurs (Hauc, 2009 : 75-81). Décrite dans son rapport à l'homme, détenteur du pouvoir social et sexuel, la femme refuse d'être un simple objet du désir masculin et, vise, parfois, à devenir indépendante (Bi-Bi dans le conte homonyme, la comtesse de Norval dans Le Quart d'heure d'une jolie femme, la marquise de Sarmé dans le roman Le Colporteur). Donc, la présence de femmes de caractère est une composante commune aux écrits libertins de Chevrier et des auteurs libertins du siècle :

Une femme de bonne Compagnie est une de ces petites Maîtresses, dont les propos sont marqués par des impertinences, les actions par des écarts, les jours par des inconstances, et les nuits par des perfidies; prompte à parler, indolente à réfléchir; elle voit un homme aimable (...), le prend sans le connaître, se livre à lui sans l'aimer, et le quitte pour le rendre indiscret (...). (Chevrier, $1752: 18$ ).

Chevrier critique également l'éducation donnée aux filles dans les instituts religieux ainsi que les mœurs lascives du clergé à travers ses personnages féminins. Ses propos sont non seulement communs aux œuvres libertines qui lui sont contemporaines, mais aussi caractéristiques de la tradition des Lumières comme suggérées dans La Religieuse de Diderot (1796).

Des propos de Julie, protagoniste des Mémoires d'une honnête femme écrites par elle-même, se dégage ce que Chevrier pense: l'auteur lorrain juge l'éducation religieuse approximative non seulement parce qu'elle n'est pas adaptée aux filles, mais aussi parce qu'elle ne met pas les filles à l'abri des caprices occasionnels des religieuses qui essayent de les adultérer :

Je vous épargnerai l'ennui du détail des premières années de mon éducation; vous saurez seulement que des mains de Madame de Verman, mon aïeule, je passai dans un cloitre où je fus élevée avec cette fausse austérité qui captive la jeunesse et ne l'instruit point. Jouet perpétuel des caprices des religieuses, je me voyais tour à tour l'objet de leurs tristes complaisances ou de leurs fades plaisanteries. Haïe sans humeur, estimée sans plaisir, le couvent m'ennuyait; j'en cherchais la raison, et un mouvement secret, que je ne pouvais démêler, me disait confusément que le cloître n'était pas fait pour moi. (Chevrier, 2005 : 37)

En outre, Chevrier remet en question la famille traditionnelle et les pouvoirs coercitifs exercés par ses membres (parents ou frères) sur les filles (la protagoniste Julie et son 
amie Sophie), condamnées à renoncer à leurs désirs pour les intérêts familiaux. Encore enfermée dans le couvent, l'adolescente Julie exprime toute sa douleur en apprenant son mariage de raison imposé par sa mère :

Je savais bien que l'intérêt réglait la plupart des mariages, mais je me figurais qu'il n'y avait que les filles de Princes qui dussent sacrifier leurs goûts à la politique, et je ne pouvais croire qu'un simple Gentilhomme eût des raisons d'État qui l'obligeassent à devenir le tyran d'une jeune personne dont il devait être l'appui [...]. Devoir austère! C'est alors qu'accablée sous ton joug j'osais t'accuser de cruauté et d'injustice! Quoi, donc, me disais-je, esclave du caprice de nos parents, ne pouvons-nous sans crime nous livrer à un penchant fondé sur la raison et la vertu? Remarques inutiles! La raison est muette, quand le devoir parle : tout lui cède jusqu'à la vertu même. (Chevrier, 2005 : 38-41)

Constante dans les écrits de Chevrier, la satire piquante contre les religieux s'exprime surtout dans le roman La vie du fameux père Norbert ex capucin, ainsi que dans le roman Le Colporteur où l'auteur fait un portrait impitoyable d'un certain père Berthier, amant d'une dame, et qui porte le masque du catholique le matin et celui du libertin le soir.

Il [père Berthier] sort le matin en soutane, et il ne veut point qu'on lui reproche de dire la messe dont il a besoin, parce qu'il travaille pour le théâtre, dont il a un plus grand besoin encore. Il craint d'ailleurs qu'on ne dise de lui ce que le grand Rousseau disait de l'abbé Pèlegrin qui était dans le même cas: "Le matin catholique, et le soir idolâtre, il dîne de l'autel, et soupe du théâtre". (Chevrier, 1993 : 829)

De plus, Chevrier vise à mettre en lumière non seulement la formation religieuse réservée aux filles, mais aussi l'éducation dispensée par des mères superficielles et qui sont la cause principale du libertinage de leurs filles : «[...] j'ai toujours vu depuis que les trois quarts des filles qui succombent, ne se livrent au libertinage que par une complaisance excessive des mères trop faciles et trop peu attentives à l'éducation de leurs enfants " (Chevrier, 1752 : 71). Ainsi, dans ses satires, Chevrier tourne en dérision les filles de l'Opéra issues des classes sociales modestes et privées de liens affectifs et de talent. Elles obtiennent toutefois du succès grâce à leurs charmes qui les aident à devenir les maîtresses d'hommes aisés et influents.

Une fille sans parents, sans amis, sans talents, n'a d'asile que celui de l'opéra ; il suffit qu'elle soit belle, pour être présentée aux yeux avides du public; [...] elle arrive sur le Théâtre pour garder la scène, elle y reste deux heures sans rien dire, et sort comblée d'éloges. Croirait-on qu'en France une femme pût réussir sans parler? Eh oui! la beauté n'est faite que pour opérer des miracles. Cette Actrice muette fixe les regards d'un jeune Etranger, ou d'un vieux Financier; on lui dit qu'elle plaît, elle le croit; on lui propose de se donner à bail pour quelque mois, elle y consent; on discute, on marchande, le prix une fois réglé, elle s'annonce comme une Demoiselle entretenue, elle ne sort que dans l'équipage de Monsieur; et voilà la célébrité décidée pour l'amant et pour elle (...). (Chevrier, 1752 : 65-66)

Ce type de personnages est un poncif de la littérature libertine.

\section{L'espace des libertins}

Les rencontres libertines décrites par Chevrier se déroulent dans des endroits caractéristiques, voués aux plaisirs de la haute société française de l'époque. Du reste, la même résidence aristocratique, l'hôtel, est symptomatique non seulement de la partition des espaces et des rapports sociaux, mais aussi conjugaux puisqu'à cette époque, la mode était aux appartements privés pour chacun des époux.

Dans la littérature libertine, les espaces "sociaux» s'opposent aux espaces privés constitués de chambres ou de recoins de la maison où les individus peuvent passer outre leurs obligations conjugales. L'intimité des boudoirs permet de trouver un refuge à l'abri de la vie mondaine des salons. C'est, en effet, au début du dix-huitième siècle, 
qu'émerge une tendance croissante à réserver des espaces privés, dans la haute société, aux femmes. Auparavant, celles-ci ne disposaient que d'un boudoir réservé à quelques activités comme la lecture, l'étude et la méditation.

De tous les espaces typiques de la littérature libertine, Paris occupe une place à part. La capitale Paris était non seulement le décor privilégié des aventures libertines des personnages, mais aussi un lieu d'initiation des libertins.

D'après Chevrier : "il faut vivre à Paris pour juger jusqu'à quel point la corruption a fait des progrès dans cette république» (Chevrier, 1752: 64-65). Julie, des Mémoires d'une honnête femme, craint les conséquences de son déplacement à Paris, considérée comme la ville des tentations. Dans les Amusements des dames de $B^{* * *}$, Chevrier décrit l'immoralité de Paris fréquentée par des femmes assoiffées de pouvoir (en particulier les filles de l'Opéra) et des hommes aux comportements ridicules.

Ces quelques réflexions sur le style de Chevrier nous permettent de conclure sur l'importance de ses écrits libertins, même si la plupart d'entre eux ont souvent suscité des scandales et la condamnation de l'opinion publique à cause de la dérision avec laquelle il a traité la monarchie et l'église.

Du point de vue du parcours littéraire de Chevrier, il faut constater que les premières œuvres libertines subissent les influences caractéristiques du dix-huitième siècle, telles que la présence des fées, la prédilection pour les éléments magiques et les riches scenari orientaux, les auteurs libertins reconnus (Claude de Crébillon et Duclos en particulier).

En effet, la production libertine de jeunesse porte de nombreux hommages à Crébillon :

M. Desmahis, jeune Auteur, dont la diction mérite des éloges, en a essayé un nouveau portrait dans des vers détachés qu'il a fait réciter à la Comédie Française, sur différents ridicules; mais $M$. de Crébillon l'a peinte avant eux, et je crois que la vérité du tableau doit ramener le public aux idées de cet Auteur ingénieux. (Chevrier, 1752 : 15)

À la différence de ses écrits de jeunesse, les œuvres ultérieures témoignent d'une indépendance par rapport à ses maîtres, en même temps qu'elles se focalisent sur des personnages comme les hommes débauchés et les femmes lascives inspirés d'une certaine France de l'époque.

\section{BIBLIOGRAPHIE}

Année littéraire (L'), novembre 1754. Consulté sur le site : https://books.google.com/books? isbn $=2862723258$

CHEVRIER Francois-Antoine, Les Ridicules du siècle, Londres [Paris, Mérigot], 1752.

CHEVRIER François-Antoine, Le Colporteur, Londres, Jean Nourse, 1762. Réédité en 1914, avec un supplément de Van Bever Adolphe, à Paris, Bibliothèque des Curieux.

CHEVRIER Francois-Antoine, Observations sur le théâtre, dans lesquelles on examine avec impartialité l'état actuel des Spectacles de Paris, Paris, Debure Le Jeune, 1755.

CHEVRIER Francois-Antoine, Recueil de ces dames [1745], dans CEuvres badines complètes du Comte de Caylus, tome XI, Amsterdam, Paris, Visse, 1787. 
CHEVRIER Francois-Antoine, Mémoires d'une honnête femme écrits par elle-même [1753], Publications de l'Université de Saint-Étienne, Saint-Étienne, 2005.

COULET Henri, « Monde et libertinage dans le roman français du XVIII ${ }^{e}$ siècle ", dans Krauss, Baher Werner (dir.), Literaturgeschichte als geschichtlicher Auftrag in Memoriam Werner Krauss, Berlin. Akademie-Verlag, 1978, pp. 177-184.

COULET Henri, Le Roman jusqu'à la révolution, Paris, Armand Colin, 1991.

DELON Michel, « Préface aux Contes immoraux », dans Veysman Nicolas et Delon Michel (dir.), Contes immoraux, édition établie sous la direction de, Paris, Laffont, 2009, pp. 7-27.

DÉMORIS René, « Les fêtes galantes chez Watteau et dans le roman contemporain », in Dixhuitième siècle, III (1971), pp. 337-357.

DIDIER Béatrice, «L'Exotisme et la mise en question du système familial et moral dans le roman à la fin du XVIII ${ }^{\mathrm{e}}$ siècle, Beckford, Sade, Potocki », in Studies on Voltaire, 152 (1976), pp. 571-586.

DIDEROT Denis, Sur les femmes [1772], in Euvres complètes, Tome X, Paris, Le club français du livre, 1971.

DUCLOS Charles Pinot, Mémoires secrets sur le règne de Louis XIV, La régence, et le règne de Louis XV [1791], dans Euvres, Tome III, Paris, Belin, 1802.

ELIAS Norbert, La società di corte, Bologna, Il Mulino, 1980.

FAVART Charles Simon, Mémoires et correspondance littéraires, Tome II, Paris, Collin, 1808.

GREEN Frederick Charles, La peinture des mours de la bonne société dans le roman français de 1715 à 1761, Paris, P.U.F., 1924.

HAUC Jean-Claude, « François-Antoine Chevrier », dans Aventuriers et libertins au siècle des Lumières, Paris, Éditions de Paris, 2009, pp. 75-81.

LACLOS Pierre-Ambroise-François, Des femmes et de leur éducation [1783], Paris, Mille et Une Nuit, 2000.

LAROCH Philippe, Petits-maîtres et roués : Évolution de la notion de libertinage dans le roman français du XVIIIe siècle, Québec, Presses de l'université de Laval, 1979.

MONTANDON Alain, Le roman au XVIIIe siècle en Europe, PUF, 1999. PONZETTO Valentina, Musset ou la nostalgie libertine, Librairie Droz, 2007.

RUSTIN Jacques, « Définition et explicitation du roman libertin des Lumières ", dans Travaux de linguistique et de littérature, Paris, Klincksieck, vol. XVI, n² 2, 1978, p. 27-34.

WALD LASOWSKI Patrick, « Préface », dans Wald Lasowski Patrick (dir.), Romanciers libertins du XVIII siècle, Gallimard, Bibliothèque de la Pléiade, Tome I, 2000, pp. IX-LX.

WEIL Françoise, Dictionnaire des journalists, -journalistes.gazettes18e.fr/journaliste/176-francoisde-chevrier WYNGAARD Amy, « Libertine space. Anonymous crowds, secret chambers and urban corruption in Rétif de La Bretonne », in Eighteenth-Century Life, 22, 2 (1998), pp. 104-122.

\section{NOTES}

1. «On dit que leurs Hautes Puissances, ne pouvant se dispenser d'acquiescer à la demande qui leur avait été faite, mais ne voulant point en même temps déroger aux privilèges de la liberté de l'Hollande, ont trouvé le moyen de concilier les choses, en expédiant à M. Chevrier, un passeport 
pour l'autre monde. On assure qu'il a été empoisonné dans un plat d'épinards, et qu'il est tombé roide mort, au moment qu'on est venu l'arrêter pour le livrer à l'ambassadeur de France.

2. «Crébillon est moins un romancier libertin, qu'un romancier qui peint des libertins «(H. Coulet (1967/1968: 365).

3. "Nous nommerons donc petits-maitres les libertins dont la seule ambition est de se faire admirer par les dames puis de les séduire par vanité, et roués ceux qui cherchent à se venger sur toutes les femmes d"une infidélité qui les a mortifiés, en menant leurs intrigues à grand bruit. Aux premiers nous associerons aussi les "apprentis" libertins qui découvrent dans l"amour-goût" une activité sociale et sentimentale à michemin de la passion et de la sensualité » (Laroch, 1979 :3).

4. L'orthographe d'origine a été actualisée.

\section{RÉSUMÉS}

François-Antoine Chevrier (1721-1762) a été oublié par la critique et le public pendant ces deux derniers siècles. Une disgrâce que pourraient expliquer, en partie, ses incessantes polémiques, médisances et autres satires. En fait, Chevrier mérite plus de considération non seulement parce qu'il est l'auteur de nombreux écrits qui s'inscrivent pleinement dans la tradition libertine du dix-huitième siècle, mais aussi parce qu'il témoigne de la situation politique décadente et du dérèglement général des mœurs de son époque. Mais son importance a été également diminuée par la renommée d'autres romanciers libertins de son siècle (Crébillon fils, Duclos, Laclos, etc.) dont il partage pourtant l'engagement personnel et les sujets traités. Le but de cette étude est de mettre l'accent sur les éléments littéraires caractérisant le style de Chevrier par rapport à la littérature libertine du dix-huitième siècle.

François-Antoine Chevrier (1721-1762) has been neglected by critics and public for more than two centuries because of his polemic and biting satires. In fact, Chevrier deserves more consideration not only because of his important production of literary works, which belong squarely in the libertine tradition of the eighteenth century, but also because he offers a proof of the decadent political situation and general habits of corruption. His importance has been however obscured by the fame of other writers of the Eighteenth century (such as Crébillon, Duclos, Laclos, etc.) with whom he shares personal engagement and subjects. So the goal of this study is to focus on the literary elements characterising Chevrier's style, compared to the rest of the libertine literary production.

\section{INDEX}

Mots-clés : Chevrier (François-Antoine), style, XVIIIe siècle, libertinage, théâtre, Ancien Régime Keywords : Chevrier (François-Antoine), style, 18th century, libertine, theatre, old Regime

\section{AUTEUR}

\section{LUISA MESSINA}

Université de Palerme Italie 